\title{
Identidad profesional de una psicóloga educacional: un estudio de caso en Chile
}

Professional identity of an educational psychologist: a case study in Chile

Identidade profissional de um psicólogo educacional: um estudo de caso no Chile

\author{
Claudia Carrasco Aguilar ${ }^{1}$ \\ María Julia Baltar de Andrade ${ }^{2}$ \\ Natalia Bastidas ${ }^{3}$ \\ Javiera López de Aréchaga ${ }^{4}$ \\ Macarena Morales ${ }^{5}$ \\ Verónica López ${ }^{6}$
}

Recibido: 18 de agosto de 2018 Aprobado: 28 de noviembre de 2018 Publicado: 21 de enero de 2019

Cómo citar este artículo:

Carrasco Aguilar, C., Baltar de Andrade, M.J., Bastidas, N., López de Aréchaga, J., Morales, M. y López, V. (2019). Identidad profesional de una psicóloga educacional: un estudio de caso en Chile. Pensando Psicología, 15(25), 1-27. DOI: https://doi.org/10.16925/2382-3984.2019.01.01

Artículo de investigación. https://doi.org/10.16925/2382-3984.2019.01.01

Fuente de financiamiento: PIA CONICYT 160009

1 Universidad de Playa Ancha

ORCID: https://orcid.org/0000-0002-8768-2440

Correo electrónico: claudia.carrasco@upla.cl

2 Pontificia Universidad Católica de Valparaíso ORCID: https://orcid.org/0000-0002-1325-6473

3 Pontificia Universidad Católica de Valparaíso ORCID: https://orcid.org/0000-0002-8419-0088

4 Pontificia Universidad Católica de Valparaíso ORCID: https://orcid.org/0000-0002-8072-8848

5 Pontificia Universidad Católica de Valparaíso Centro de Investigación para la Educación Inclusiva ORCID: https://orcid.org/0000-0002-1624-4873

6 Pontificia Universidad Católica de Valparaíso Centro de Investigación para la Educación Inclusiva ORCID: https://orcid.org/0000-0001-7405-3859 


\title{
Resumen
}

Introducción: La psicología educacional enfrenta la necesidad de delimitar acciones y roles, superar la atención de las diferencias individuales, y considerar el papel de la cultura escolar. Estos desafíos impactan en la identidad de psicólogas y psicólogos educacionales, actualmente en su mayoría contratados bajo la Ley SEP en Chile.

Objetivos: Esta investigación analizó la identidad de una psicóloga escolar y los procesos de interacción social que la llevan a construir esta identidad, en el contexto de una escuela municipal chilena con sus respectivas dinámicas micro políticas en el marco de la Ley SEP.

Metodología: Se realiza un estudio de caso único a través de entrevistas en profundidad, un grupo focal, con una psicóloga contratada con recursos de la Ley SEP, docentes y estudiantes. Se realiza análisis de contenido categorial.

Resultados: Existe una tensión de la psicóloga entre ser y no ser de la escuela, así como un posicionamiento periférico. Se muestra una identidad tensionada bajo un proceso de negociación de la identidad profesional. Conclusiones: Los resultados se discuten en torno a la intensificación y desarticulación del trabajo, la complicidad ideológica, el rol clínico de la psicología, y el impacto de la Ley SEP y de la micro política en este escenario.

Palabras clave: Chile, identidad profesional, psicología educacional.

\begin{abstract}
Introduction: Educational psychology faces the need to delimit actions and roles, overcome catering for individual differences, and consider the role of school culture. These challenges impact on the identity of educational psychologists, who are currently being hired under the SEP Law in Chile.

Objectives: This study described the identity of a school psychologist and the processes of social interaction that lead her to construct this identity, in the context of a Chilean municipal school with its respective micro-political dynamics within the framework of the SEP Law.
\end{abstract}

Methodology: A single case study is conducted through in-depth interviews and one focus group, with a psychologist hired with resources from the SEP Law, teachers and students. Categorical content analyses was performed.

Results: There is a tension of the psychologist between being and not being part of the school, as well as peripheral positioning. Her identity is put into tension under a process of negotiation of professional identity.

Conclusions: The results are discussed concerning the intensification and disarticulation of work, the ideological complicity, the clinical role of psychology, and the impact of the SEP Law and the micro politics in this scenario.

Keywords: Chile, professional identity y School psychology

\section{Resumo}

Introdução: A psicologia educacional enfrenta a necessidade de delimitar ações e papéis, superar a atenção das diferenças individuais e definir o papel da cultura escolar. Esses desafios impactam a identidade de psicólogos escolares, atualmente contratados principalmente pela Lei SEP no Chile.

Objetivos: Esta pesquisa analisou a identidade de um psicólogo escolar e os processos de interação social que a levaram a construir essa identidade, no contexto de uma escola municipal chilena com suas respectivas dinâmicas micropolíticas no marco da Lei SEP. 
Metodologia: Este é um estudo de caso único com entrevistas em profundidade, um grupo focal, com um psicólogo contratado com recursos da Lei SEP, professores e alunos. Nós fizemos uma análise de conteúdo com categorias.

Resultados: Existe uma tensão do psicólogo entre ser e não ser da escola, bem como um posicionamento periférico. Uma identidade estressada é mostrada sob um processo de negociação de identidade profissional. Conclusões: Os resultados são discutidos em torno da intensificação e desarticulação do trabalho, da cumplicidade ideológica, do papel clínico da psicologia e do impacto da Lei SEP e da micropolítica nesse cenário.

Palavras-chave: Chile, identidade profissional, psicologia educacional.

\section{Introduction}

\section{Algunos desafíos para la psicología educacional}

De forma similar a gran parte de Occidente, en Chile la psicología en educación surge como una manera de estudiar los procesos escolares, avanzando hacia la búsqueda de soluciones a los problemas derivados de estos procesos (Baltar y Carrasco, 2013). Desde entonces ha sido un verdadero desafío delimitar las acciones que los profesionales de esta disciplina deben llevar a cabo en las escuelas, pues muchas veces se presenta una gran diversidad de roles (González, González y Vicencio, 2014; Ossa 2011). Diversos autores cuestionan que el rol de la psicología se haya focalizado durante décadas, casi exclusivamente, en la atención de las diferencias individuales, ya sea porque de este modo se invisibiliza el papel del contexto escolar y las relaciones que dan origen a las diferencias individuales que se vuelven conflictivas (López, Carrasco, Morales y Ayala, 2011; López y Carrasco, 2018), o por los procesos de rotulación a los que se ha contribuido al patologizar estas diferencias (Bahía Bock, 2003; Baltar, 2003; Fernández; Vázquez y Ubiarco, 2016). Para Baltar y Carrasco (2013) esto da cuenta de un quehacer y de un rol que no puede ser llamado propiamente "educacional", debido a que remite casi exclusivamente a los procesos escolares.

El contexto educativo tiene sus propias particularidades. La cultura escolar muchas veces es obviada por los profesionales de la psicología que trabajan en centros educativos, y esto tiende a generar consecuencias que debilitan su trabajo y limitan sus acciones (Juliá, 2006). No siempre se puede establecer un trabajo colaborativo con otros actores educativos, y muchas veces se desarrollan luchas de poder en torno a las funciones a desarrollar. Así es como diferentes psicólogos y psicólogas en educación tienen dificultades para delimitar su trabajo en relación con otros profesionales de la educación. Incluso desde los enfoques más clásicos, que ubican esta disciplina en el campo de las diferencias individuales, y desde el diagnóstico clínico han existido 
debates y disputas por delimitar funciones. Un ejemplo de esto lo expone Pérez (2016) en España, al concluir que, si bien la evaluación psicológica en el ámbito educativo "es una función fundamental del psicólogo y no de cualquier otro profesional docente o no" (p. 475), a los servicios de orientación públicos españoles estarían "accediendo profesionales de distintos perfiles que bajo la denominación de 'orientadores' hacen funciones propias del psicólogo educativo" (p. 475). La autora termina indicando que es necesario un cambio en la legislación para que esta práctica se regularice.

Asimismo, existen estudios que muestran que esta dificultad de trabajar colaborativamente implica un encapsulamiento de los psicólogos y psicólogas (López, et al., 2011), alejamiento del currículum y los procesos educativos (De Souza, Ribeiro y Cintra da Silva, 2011; García, Carrasco, Mendoza y Pérez, 2012), y el enfrentamiento ambiguo ante diferentes expectativas (Compagnucci y Cardós, 2009; González, et al., 2014).

Frente a estas dificultades y tensiones, al preguntarse por la identidad de psicólogos y psicólogas educacionales, López y Carrasco (2018) concluyen que la diferencia entre un profesional de la psicología en un contexto escolar y un psicólogo educacional es la aproximación y el trabajo con el curriculum. Para las autoras, es necesario establecer un diálogo auténtico con la pedagogía, hasta encontrar entre ambas el intersticio que se enmarca en la educación como ciencia social. Las autoras hacen un llamado a la psicología educacional para convertirse en una disciplina que utilice el desarrollo científico de la psicología en los procesos de enseñanza y aprendizaje, pero no en un sentido tradicional de apoyo al docente para hacer efectivo el aprendizaje del estudiantado, sino para comprender las acciones de carácter psicosocial en un marco propiamente educativo. De todos modos, este trabajo con el curriculum no puede ocurrir al margen de los contextos específicos en los que se desarrolla la vida escolar. Autores como Vera-Bachmann, Valenzuela y Álvarez-Espinoza (2016) invitan a considerar todo tipo de factores en estos desafíos, poniendo atención en determinados contextos como el área rural, en los cuales sería aún más compleja esta labor. Es por ello que un aspecto crucial del quehacer de la psicología educativa será la visión a partir de la propia complejidad de cada establecimiento (Barraza, 2015), considerando además el masivo aumento de psicólogos y psicólogas en los centros educativos que ha vivido Chile en los últimos diez años (Vera-Bachman, y Salvo, 2016).

\section{La Ley de Subvención Escolar Preferencial (Ley SEP) como política educacional}

Como parte de un conjunto de iniciativas orientadas al mejoramiento tanto de la calidad como de la equidad de la educación chilena, los actuales gobiernos han 
desarrollado diversos programas dentro de los cuales se han focalizado en procesos y resultados de aprendizaje, y en las condiciones estructurales en las cuales se desarrolla el trabajo educativo. El énfasis en lograr la equidad se realiza atendiendo a las diferencias y en la discriminación positiva de los más vulnerables (Ministerio de Educación, 2009). Una de estas iniciativas consistió, desde el año 2009, en la Ley de Subvención Escolar Preferencial (Ley SEP). Ésta señala derechos y deberes, tanto para los sostenedores - administradores públicos y privados de los centros escolares-, como para el Mineduc (Ministerio de Educación), los que quedan establecidos en un convenio que regula su implementación. En este convenio, las escuelas se comprometen mediante la elaboración de un plan de mejoramiento a destinar acciones orientadas al estudiantado prioritario en las áreas de gestión curricular, gestión directiva y de liderazgo, gestión de la convivencia escolar y gestión de los recursos (Bellei, Osses y Valenzuela, 2010). Con ello, la ley establece que cada escuela recibirá una subvención escolar complementaria a la subvención regular, por cada estudiante prioritario. Es decir, por cada estudiante con indicadores socioeconómicos de alta complejidad -en situación de vulnerabilidad socioeconómica-. En Chile, la educación pública municipal y particular subvencionada se financia a través de aportes que el Estado realiza a través de subvenciones por estudiante, según su asistencia a clases promedio mensual. Entonces, este dinero ingresa con el fin de que las escuelas generen procesos de mejoramiento orientados especialmente al desarrollo integral de estos estudiantes en riesgo social, pero la renovación está sujeta a que la escuela demuestre haber alcanzado los logros comprometidos. En caso contrario, el Mineduc tiene autoridad para revocar el reconocimiento oficial.

Esta ley ha traído consigo una serie de iniciativas por parte de los centros educativos, y dentro de éstas se encuentra un progresivo aumento de contratación de profesionales especialistas como psicólogos y psicólogas (Raczynski, Muñoz, Weinstein, y Pascual, 2013; Sandoval y Lamas, 2017). Desde la perspectiva de Sandoval y Lamas (2017), las estrategias de atención individual y directa al estudiantado que psicólogos y psicólogas realizan en el marco de sus acciones bajo la contratación con recursos de la Ley SEP, los llevan a trabajar de forma aislada del resto de los profesionales, especialmente del profesorado. Sin embargo, la ley no establece funciones ni roles a los profesionales psicosociales como psicólogos y trabajadores sociales y, de hecho, tampoco exige su contratación. La ley sólo señala que este estudiantado debe recibir apoyos psicosociales, lo que ha sido interpretado por muchos colegios como la necesidad de contratar profesionales que brinden atención directa.

La Ley SEP cuenta con diferentes críticas y cuestionamientos en varios niveles. Bellei y García Huidobro (2006) manifiestan que se basa en el castigo y la recompensa. 
Estas medidas están sujetas a los resultados obtenidos por las instituciones educativas en la prueba estandarizada que evalúa los resultados de aprendizaje del estudiantado en Chile, denominada Sistema de Medición de Calidad de la Educación (Simce). Esta consiste en una prueba censal y con altas consecuencias, ya que con sistemáticos bajos resultados incluso se puede perder el reconocimiento oficial del Ministerio de Educación y cerrar la escuela. Para los autores, la propuesta de la ley es en parte una estrategia de política educativa más general en donde se asocian sanciones e incentivos a los actores educativos, dependiendo del resultado que obtengan sus estudiantes. Esta lógica implica que aquellas escuelas que no cumplan con los resultados esperados serán sancionadas con la disminución o corte de la subvención corriendo el riesgo de cerrar el establecimiento. Estos mecanismos han sido denominados, por autores como Assaél, Contreras, Corbalán, Palma, Campos, Sisto y Redondo (2012), como "dispositivos de vigilancia".

Estos dispositivos generan en la escuela una dinámica de constante presión tanto para la propia escuela como para la organización y quienes la conforman. Finalmente, tal como señalan estos autores con respecto a los efectos de la Ley SEP en la escuela, pareciera que se ha convertido paulatinamente a la cultura escolar en una cultura de auditoría (Assaél et al., 2012). Bajo esta lógica, los actores educativos se ven en la obligación de producir constante evidencia, de acuerdo a planes y metas, llevando a una intensificación del trabajo (Hargreaves, 1998). Todo esto conduce a que se desarrolle solamente lo que es estrictamente necesario (Hypólito, Vieira y Pizzi, 2009). La cultura de la auditoría se entremezcla así con la propia cultura escolar, la que a su vez está cargada de diferentes relaciones micropolíticas (Assaél, Acuña, Contreras, y Corbalán, 2014). En definitiva, las acciones de psicólogos y psicólogas en escuelas enmarcados en la Ley SEP, estarían sujetas a las presiones propias de la cultura de la auditoría, a la vez que tendrían un foco psicosocial, sin que se haya definido previamente lo que esto significa. Así también, los centros educativos sólo recientemente han comenzado a contratar a estos profesionales a través del Código del Trabajo. Hasta hace poco tiempo, cada colegio podía definir el medio de contratación de estos profesionales, y muchos se encontraban trabajando de forma autónoma en las escuelas, aunque deban cumplir horarios entre otras cosas. La contratación en modalidad honorarios le ahorra al empleador el pago de los seguros sociales y, por lo tanto, impacta en la relación costo-beneficio que instala las políticas de voucher (Verger, Bonal y Zancajo, 2016). 


\section{Micro política en la Escuela como elemento relevante del contexto organizacional}

Los actores educativos son actores políticos, sumergidos en la complejidad de la escuela, en la que se hallan diversos estamentos que son colectividades agrupadas bajo lógicas e intereses en común (Bardisa, 1997). Estas personas emplean estrategias para posicionarse, colaborar, acceder a recursos, aliarse o comprometer a otras personas para conseguir sus propósitos. Es así como es posible definir a las instituciones escolares como si se trataran de campos de batalla en los cuales se desarrollan permanentes conflictos y luchas de poder que terminan por promover cambios institucionales (Ball, 1994).

Para algunos autores, la Ley SEP es una iniciativa que ha conseguido equilibrar tanto la macro como la micropolítica de los centros escolares (Raczynski, et al., 2013). Sin embargo, las dinámicas micropolíticas de la escuela traen consigo mecanismos de dominación e ideologías presentes en la sociedad, así como relaciones políticas y económicas que permitirían comprender cómo es la escuela. Es así como, en palabras de González (1990, citado en Bardisa, 1997), se entiende que "la escuela desempeña, a través de sus prácticas y relaciones, un papel ideológico dentro del contexto sociocultural en el que está inmersa" (p. 19). Las dinámicas micropolíticas de las instituciones educativas se pueden evidenciar por medio de sus diferentes fuerzas de lucha que enfrentan aquello que está instituido, así como lo instituyente. Esto ocurre por medio de diferentes luchas por el poder, diversidad de metas, intereses particulares, estrategias y rivalidades por el control, entre otras situaciones (Gómez, 2010). Para participar de estas luchas de poder, las personas se organizan en grupos políticos y grupos de interés. Éstos emergen cuando hay objetivos comunes, por lo que se diseñan estrategias de intercambio de recursos por influencias de quienes tienen el poder y toman las decisiones al interior de las escuelas. Así es como se imponen las lógicas de acción (Bardisa, 1997). El análisis de la micropolítica resulta relevante en este caso debido a que frente la ausencia de un marco global que declare funciones o roles de psicólogos y psicólogas contratados bajo la Ley SEP, serán las formas de interactuar en cada centro educativo las que determinen estas funciones. Sin embargo, la autonomía de los centros no es absoluta en esta materia. Esta autonomía es relativa, pues dependen del exterior para trabajar y tener recursos (Zibas, 1997). Esto configura a los recursos como fuente de poder y control en la escuela, pues moviliza a conseguir alianzas y compromisos, produce afinidades o luchas entre grupos para obtener los tan codiciados recursos educativos. Con la llegada de diversos especialistas a la escuela, se dificultan las planificaciones, y al aumentar la cantidad de reuniones, las instancias de trabajo con los nuevos profesionales se transforman en una suerte de voluntariado. 
No es sólo la falta de tiempo lo que afecta el interés en participar de estas instancias, sino que además hay una reacción por parte del profesorado ante propuestas de mejoramiento heterogéneas y que dependen de la edad, sexo y experiencias de vida de los docentes que llevan trabajando en las instituciones por mucho tiempo (Bardisa, 1997). Además, el estilo de dirección y liderazgo influye de sobremanera en las relaciones micropolíticas tanto con estos profesionales de apoyo como dentro del propio profesorado, instala discusiones en torno a la participación, solidaridad, comunidad y democracia en la escuela (Fuentes y Cruz, 2016; Gómez, 2010; Rodríguez, 2016).

Otro factor que dificulta el trabajo con los profesionales de apoyo en la escuela es la cultura del individualismo docente. En ésta, profesionales son excluidos o se autoexcluyen de las decisiones de la escuela, y el aula se convierte en el centro, quedando el resto de las personas de la escuela fuera de los procesos pedagógicos que suceden. A esto, Bardisa (1997) le llama "celularismo", y da cuenta del aislamiento que dificulta la colaboración entre colegas y el proceso de aprendizaje colaborativo, pues señala que los profesores, sobre todo los más antiguos, son quienes parecieran más reacios al cambio.

Todo lo anterior configura, paradójicamente, el escenario de la complicidad ideológica entre psicología y educación, colaborando ambas con procesos de rotulación, estigmatización y externalización de responsabilidades. A pesar del aparente distanciamiento entre ambas disciplinas, convergen en este escenario educativo con un fin en común del cual no son conscientes. La psicología, como disciplina, sería cómplice de la educación en el señalamiento del "culpable", a través de la acumulación de evidencia científica positivista que, de la mano de la psicología del desarrollo, habría instalado en las escuelas la creencia de que los problemas de adaptación y aprendizaje son responsabilidad fundamental del niño, de la niña y su familia. Esto implicaría la naturalización de las etapas del desarrollo como una trayectoria lineal e individual, y su relación con los procesos de aprendizaje como un dogma cultural en la escuela, que convierte a la institución escolar en un espacio inflexible y resistente al cambio, con dificultades para observar su propia responsabilidad en los aprendizajes del estudiantado (Bahía Bock, 2003). Así es como se explica que, frente a la inexistencia de definición de funciones, exista una tendencia a confirmar el rol clínico de la psicología en la escuela.

Si bien pareciera ser que la micropolítica distancia al profesorado de otros profesionales, la cultura escolar termina por confirmar que el enfrentamiento de grupos de poder se resuelve a través de espacios de fuga que ubican al estudiantado como el gran responsable de los conflictos de la escuela. Estas formas particulares de llevar a cabo el trabajo de la psicología en un contexto altamente complejo como es la 
escuela, impactan de forma significativa en la identidad de estos profesionales, así como en sus acciones cotidianas. Esta identidad no sólo se condiciona a partir de esta cultura micropolítica sino que, además, se ve impactada por las macropolíticas sociales, en este caso, la Ley SEP. Analizar el contexto macropolítico es una oportunidad para explicar diferentes lógicas de acción que construyen los grupos de interés desde fuera de la institución escolar, los que inevitablemente ingresan e influyen en la escuela (Bardisa, 1997). Estos grupos externos a las escuelas pueden vehiculizar sus acciones e influencias a través de políticas públicas. Es por ello que las políticas nacionales e internacionales generan cierta forma de interactuar en los centros educativos, y tienen consecuencias sobre los diferentes actores que allí se desempeñan. Las políticas públicas ofrecen identidad colectiva e individual, ya que las personas quedan sujetas, al menos parcialmente, a éstas (Ibáñez, 1994).

\section{Identidad profesional: entre lo micro y lo macro}

Al analizar el constructo general de identidad es posible distinguir una dimensión experiencial que se da en un contexto de relaciones sociales permitiendo dos procesos simultáneos: 1) la identificación con otros, y 2) la diferenciación de ellos (Íñiguez, 2001; Tajfel y Turner, 1979). Este doble proceso se da en un marco micro, meso y macro político y social. En el nivel micro se desarrollan los procesos de subjetivación y representación del otro - personal y grupalmente-. En el nivel meso, se desarroIla la identidad colectiva a partir de dinámicas e interacciones culturales. En el nivel macro se desarrollan todos los aspectos políticos, ideológicos y de ordenamiento mundial que influyen sobre los procesos de construcción de identidad, como las políticas neoliberales, y las grandes ideologías nacionales (Íñiguez, 2001; Palacio, Correa, Díaz y Jiménez, 2003).

Los niveles micro y meso se conectan entre sí, el primero requiere de la intersubjetividad, y el segundo le impone a la identidad la necesidad de confrontarse permanentemente con otras identidades como parte de las dinámicas sociales. Cuando se consideran los niveles micro y meso, aparece la identidad social, definida como el sentimiento de pertenecer a un grupo o colectivo mayor (Palacio et al., 2003). Las diferentes imágenes ofrecidas y recibidas generan una identidad que permite a las personas convertirse en observadoras de sí mismas, permitiendo hacer reajustes, modificaciones o cambios. Por ello es posible concluir que la identidad depende del contexto en el que tiene lugar una interacción, así como del modo en como las personas negocian el significado que le darán al contexto. Esto se denomina identidad situada (Íñiguez, 2001). Al utilizar el nivel macro como categoría de análisis, la identidad 
individual o personal aparece definida como una acción doble, ya que sería producto tanto de la sociedad como del propio individuo, para lo cual debe considerar tanto el contexto histórico donde surge como el marco político de éste (Pujal, 1996).

Desde esta perspectiva, los fenómenos identitarios no pueden ser considerados sólo de origen psicológico o comportamental, y deben ser analizados como construcciones situadas históricamente y emergentes en los procesos sociales (Pujal, 1996). En este contexto, si se define la identidad como una construcción subjetiva y una inscripción social, es posible distinguir dos dimensiones de la misma: "identidad para sí" e "identidad para otros" (Dubar, 2000 citado en Bolívar y Ritacco, 2016). La identidad para sí responde a los significados sobre el sí mismo que se derivan de un proceso biográfico, mientras que la identidad para otros se corresponde a la dimensión social y relacional que busca constantemente el reconocimiento de los demás. "Este proceso de construcción identitaria se juega como una transacción entre la identidad atribuida y la identidad aceptada por el individuo concreto" (Bolívar y Ritacco, 2016, p. 165). La identidad profesional, en el contexto escolar, implica la negociación de identidades no sólo en relación con las expectativas, sino también con las imágenes que los actores educativos reciben sobre sí (Bolívar y Ritacco, 2016).

Esta negociación de la identidad profesional en la escuela no sólo afecta a directores y docentes, sino a todos quienes se desempeñan en ella. Diferentes estudios han concluido que la Ley de Subvención Escolar Preferencial está modificando las interacciones en las escuelas e incluso se encuentra asignando nuevas identidades al profesorado (Rojas, y Leyton, 2014), a los administradores educacionales (DonosoDíaz, y Benavides-Moreno, 2017) y a los propios centros educativos (Castro, Mazo y Quintanilla, 2016), a través de la instalación de las nociones de vulnerabilidad escolar y gerenciamiento educativo que esta normativa -y otras - lleva de la mano (Ligueño, Palacios, y Sandoval, 2018).

¿Cómo se construye entonces, la identidad de un profesional de la psicología en este contexto macro y micro político escolar? Para responder a esta pregunta, a continuación se presentan los resultados de un estudio que describió la identidad de una psicóloga escolar, y los procesos de interacción social que la llevan a construir esta identidad, en el contexto de una escuela municipal chilena con sus respectivas dinámicas micropolíticas en el marco de la Ley SEP. 


\section{Método}

\section{Diseño}

El presente estudio corresponde al paradigma cualitativo, desde una perspectiva interpretativa (Quintana, 2006). El diseño es un estudio de caso único, (Rodríguez, Gil, y García, 1996) que apuntó a la descripción y análisis en profundidad de un fenómeno educativo. Este caso único se trata a la vez de un caso típico, descrito por Quintana (2006) como aquél que tiene como objetivo mostrar - a quién no está familiarizado con la realidad objeto de análisis - los rasgos más comunes de dicha realidad. Por ello, para la elección del caso, se seleccionó el contexto político -en este caso la Ley SEP- debido a que es el contexto que está dando gran posibilidad de contratación a los profesionales de la psicología actualmente en Chile. En este escenario, se eligió una escuela municipal que contrató a una psicóloga luego de implementada la ley a través de los recursos otorgados por la misma sin tener una descripción del cargo, pese haber solicitado a la psicóloga un apoyo psicosocial. Se trata de una escuela básica-primaria, con una matrícula que oscila entre los 200 y 250 estudiantes como muchas otras escuelas del país, ubicada en una zona urbana, en la cual trabajan dos psicólogas, una de ellas contratada con los recursos de esta ley, y la otra, con recursos asociados a un decreto para atender a estudiantes con necesidades educativas especiales. Como suele ocurrir en el caso de muchos establecimientos educativos, la psicóloga fue negociando paulatinamente su rol y funciones.

\section{Participantes}

Para la selección de las personas participantes se realizó un muestreo intencionado (Salamanca y Martín-Crespo, 2007), debido a las necesidades de información que se debe producir con actores claves para arrojar los resultados más importantes. Estos actores corresponden a una psicóloga contratada desde el año 2010, el director de la escuela desde 2010 y la jefa de UTP, quien está a cargo del plan de mejora. Posteriormente, se procedió a un muestreo de avalancha, en el que se solicitó a los informantes clave que recomendaran a posibles participantes, lo que es más práctico y eficiente, posibilitando la relación de confianza con los nuevos participantes y la posibilidad de acceder a quienes son difíciles de identificar (Salamanca y MartinCrespo, 2007). Estos actores corresponden a dos docentes, una profesora jefa de un primero básico y una profesora jefa de un sexto básico, siete estudiantes de tercero y cuarto básico que han participado en talleres grupales implementados por la psicóloga, y la inspectora general de la escuela, quien lleva más de tres años en el 
establecimiento. La tabla 1 muestra un resumen de los participantes asociados a la técnica de producción de información utilizada. Todas las personas entrevistadas se han vinculado laboralmente de manera directa con la psicóloga de este estudio.

La psicóloga entrevistada estudió psicología en una universidad privada de la región, tiene menos de 30 años de edad, es soltera y su formación fue mayoritariamente clínica. Sin experiencia en el área educacional, ella llegó a la escuela el año 2010 por concurso público. Para ello, presentó al director un plan de intervención que buscaba realizar acciones en el patio durante los recreos. Inicialmente fue contratada por 40 horas, ascendiendo luego a la jornada completa de 44 horas. A través de acciones lúdicas en los recreos, ella esperaba contribuir al mejoramiento del clima escolar, para lo cual contó con el apoyo del director que se encontraba liderando el colegio entonces. Posteriormente, hubo un cambio en la dirección de la escuela, y aunque un año después retornó, esto fue significado como un quiebre por la psicóloga quien desde entonces tiene como jefatura directa a la jefa de UTP y no al director. Es importante señalar que la Ley SEP no establece funciones para los profesionales de la psicología y que, aunque su contratación se ha masificado producto de esta ley, no existen lineamientos específicos ministeriales para delimitar sus funciones. Es por ello que en cada centro educativo existe libertad para establecer funciones en la medida en que de alguna manera se trabaje con estudiantes en riesgo social. En este caso en particular, la psicóloga fue negociando sus funciones, como suele ocurrir en la mayoría de los establecimientos chilenos. No es común que exista una descripción del cargo al ingreso de psicólogos y psicólogas en educación, por lo que la tendencia es que cada profesional presenta un proyecto de acción y las direcciones educativas muchas veces exigen un rol de atención clínica. Por ello, muchos profesionales contratados, sobre todo en los primeros cinco años de implementada la ley, tienen formación más clínica que educacional.

\section{Técnicas de producción y análisis de la información}

Se realizaron entrevistas en profundidad (Prieto, 2001) y un grupo focal (Canales, 2006). Las primeras, consistieron en encuentros con diferentes actores (ver tabla 1) y se focalizaron sus experiencias, emociones y sentimientos, a través de temas vinculados con las expectativas de y sobre la psicóloga, interacciones, roles, entre otros. En el caso del grupo focal, éste se realizó con estudiantes, con el fin de definir sus apreciaciones respecto de la experiencia directa de trabajo con la psicóloga. En este contexto, se siguieron las recomendaciones de Canales (2006), se focalizó una experiencia vivida, entendida como la representación o comprensión que tienen las personas. 
Las entrevistas y el grupo focal fueron trascritos de forma textual, y analizados a través del análisis de contenido categorial temático (Vázquez, 1994), el cual releva el lugar del contexto en el análisis e interpretación de la información. Para esto se realizó un preanálisis, que consideró la organización del material a analizar; luego, se realizó una codificación para transformar los datos brutos en datos útiles; y finalmente, se llevó a cabo una categorización, clasificando el material en base a criterios de diferenciación, y se concluyó con dos categorías centrales.

Tabla 1. Participantes y técnicas de producción de información

\begin{tabular}{|c|c|c|}
\hline Actores & No. de Entrevistas & Técnica de producción \\
\hline Docente de aula 1 & 1 & Entrevista \\
\hline Docente de aula 2 & 1 & Entrevista \\
\hline Director & 2 & Entrevista \\
\hline Jefa de Unidad Técnico Pedagógica (jefa de UTP) & 2 & Entrevista \\
\hline Siete Estudiantes de 3ro y 4to básico & 1 & Grupo Focal \\
\hline Psicóloga contratada por Ley SEP & 2 & Entrevista \\
\hline 1 Asistente de la educación & 1 & Entrevista \\
\hline
\end{tabular}

Fuente: elaboración propia

\section{Resultados}

Para la presentación de los resultados se han estructurado dos categorías. La primera categoría, denominada "Tensión entre ser y no ser de la escuela", da cuenta del proceso de negociación de la identidad profesional en la escuela desde las dimensiones teóricas de "identidad para sí" e "identidad para otros". Por su lado, la segunda categoría, denominada "Posicionamiento periférico de la psicóloga en la escuela", da cuenta de las interacciones que construyen esta identidad tensionada.

\section{Categoría 1. Tensión entre ser y no ser de la escuela}

La psicóloga se encuentra en una constante negociación de su identidad como profesional de la escuela, desde una "identidad para sí" y una "identidad para otros". El contexto en que se presenta esta negociación es a partir de su contratación con recursos de la Ley SEP, ya que a partir de aquello se le atribuyen funciones vinculadas con el plan de mejoramiento elaborado desde la dirección de la escuela, debe así realizar talleres para estudiantes y sus familias en el ámbito de convivencia escolar. 
La "identidad para sí" se construye como una psicóloga "de" la escuela, en cuanto esta profesional refiere sentirse parte de la institución, más allá del plan de mejoramiento, de su contrato y sus especificaciones. Se ve a sí misma como una integrante de la comunidad, y cree que su labor en la escuela ha sobrepasado los límites de los talleres y del plan de mejoramiento, realizando otras labores y acciones enmarcadas en la convivencia.

Puede ser como un mal concepto adquirido, yo, que yo no me siento la psicóloga SEP, yo me siento la psicóloga de la escuela. Y en este caso también la escuela no son los alumnos, sino que son los profesores, los apoderados, los auxiliares, los paradocentes, todos. Entonces si puedo generar un apoyo a todas las áreas, esa al final sería como mi expectativa general.

En este proceso de construcción identitaria se ponen en disputa los planos de la propia trayectoria y relacionales. Desde el relato que la psicóloga construye de sí misma en la escuela, ella se relata como una profesional que hace más de lo que está estipulado, concibiendo como un deber de la profesión atender a las demandas que considera pertenecientes al ámbito de la psicología y que le parecen satisfactorias. Esto evidencia la manera en que la identidad se corresponde tanto a una construcción subjetiva como a una inscripción social.

Pero no solamente se basa en lo que dice el papel, o sea uno como psicólogo realmente como por un tema ético muchas veces hace más de lo que está presupuestado, y yo lo hago porque me gusta.

Desde el plano relacional, existe una visión heterogénea con respecto a la figura de la psicóloga. La profesional sabe que existen algunos integrantes de la comunidad que no la ven como "la psicóloga de la escuela" y que no la identifican ni validan como tal. Éste es un nudo crítico entre los elementos relacionales y otros más personales de su construcción identitaria, pues ella manifiesta una necesidad de ser reconocida como "la psicóloga de la escuela" desmarcándose del rol de "la psicóloga SEP", buscando que los demás actores la reconozcan como un aporte a la escuela. Estas expresiones identitarias están dando cuenta de las maneras en que se relacionan los niveles micro y meso de la identidad, en cuanto subjetivación y representación. 
Hay algunas personas que todavía no me ven como la psicóloga de la escuela me ven como psicóloga SEP a diferencia de como yo me siento aquí, que yo me siento la psicóloga de la escuela.

En este contexto aparece un nivel meso social en el cual la psicóloga es puesta en un lugar de integrante de la comunidad educativa, con roles y funciones parecidas al resto de los profesionales. Sin embargo, también se genera una distancia disciplinar, que separa los ámbitos psicológicos de los pedagógicos, apareciendo una distinción de funciones o especificaciones disciplinarias en la escuela. Esto implica una posición jerárquica de quien aclara las funciones y los roles que se asignan a la psicóloga en la escuela, así como por las otras personas con las cuales interactúa. Es posible señalar entonces que la tipificación de funciones y roles implica una identificación y heteroidentificación de éstos, tal como muestra el siguiente extracto de entrevista de la jefa de UTP.

Bueno, ellos [en referencia a los psicólogos] estaban generalmente en las reuniones. Generalmente la psicóloga tiene participación en los talleres nuestros, a no ser que sea algo muy específico. Cuando es algo muy específico y ella no tiene ninguna injerencia. Esas son las únicas veces que se les dice a ellas que continúen con su trabajo y nosotros con lo nuestro, porque son psicólogos.

Esta identificación y heteroidentificación enfrenta la dimensión experiencial de la identidad con aquella que emerge del contexto de relaciones sociales, en un doble proceso que expresa más de una tensión. Estas tensiones aparecen reproducidas en algunos elementos de la propia psicóloga, quien construye un rol clínico en su quehacer enfocado a la atención de casos, basados en la realización de diagnósticos individuales del estudiantado, quienes, a su vez, validan y confirman este rol tal como muestra el segundo extracto que se presenta a continuación.

Por ejemplo, estoy acá en un taller y me dicen: —Pucha el Juanito, aquí y allá. Trato como de adecuar el espacio para ir a contenerlo.

La [nombre de la psicóloga] nos preguntaba qué juegos nos gustaba y cómo éramos, porque yo antes era malo, porque tenía muchos conflictos, problemas. Ahora no. 
Así, el nivel meso de la identidad, en este caso, estaría compuesto por las percepciones y significados de todas las personas que componen esta comunidad educativa. Estas tensiones cuestionan su pertenencia como una agente integrada en la comunidad escolar pero, al mismo tiempo, le otorgan identidad respecto de sus funciones y le permiten posicionarse dentro del discurso escolar a partir del rol clínico. En este sentido, este rol le ofrece un lugar único en la dinámica escolar pero que en términos de pertenencia es débil. Al connotar un rol clínico, la psicóloga se ubica a sí misma como una profesional de la salud que ingresa al campo educativo, encapsulando su trabajo en un conocimiento disciplinar específico que la aleja del currículum y los procesos educativos, y la enfrenta de forma ambigua frente a las múltiples expectativas que existen sobre ella. Es por esto que el discurso de una profesora entrevistada sobre el aporte que esta psicóloga genera a su práctica cotidiana es presentado de forma ambigua, siendo introducido por la palabra "supuestamente" en la oración. Lo que se cuestiona de forma implícita con el uso de esta palabra, es la pertinencia del actuar de la psicóloga, y su dominio experto sobre técnicas para trabajar con estudiantes en situación de vulnerabilidad.

Supuestamente ella sabe las técnicas como psicóloga para orientarte en el cómo trabajar con ellos

Sin embargo, ella misma señala que esa superioridad o experticia no se enmarca en una estrategia de trabajo, sino más bien, en una suerte de apoyo improvisado al docente, bajo la metáfora de "apagar incendios", lo que le confiere una identidad de apoyo a lo que cada docente vaya necesitando. De este modo, si bien la improvisación puede ser analizada como una forma de negociación de la propia identidad, es al mismo tiempo un fracaso frente a intentar usar el desarrollo científico de la psicología como apoyo para los procesos de enseñanza y aprendizaje.

Al final, el hecho de apagar incendios es algo que le acomoda a los profesores. Es como uno se siente útil también, si al final estás generando un apoyo. Realmente ves al profesor que está sobrepasado y que no tiene las herramientas necesarias para contener.

A partir de lo anterior, es posible señalar que hay diferentes elementos de la cultura escolar que no son del todo considerados en las estrategias que implementa la psicóloga, y que pueden estar a la base de la percepción del debilitamiento de su trabajo y de la limitación de sus acciones. 


\section{Categoría 2. Posicionamiento periférico de la psicóloga en la escuela}

Bajo la premisa de que la escuela es un campo de lucha y que en ella se comparten lógicas y se busca conseguir los intereses ya sean propios o comunes, en esta categoría se revisarán cuáles son las relaciones, luchas y alianzas que se dan en esta escuela en los dos ámbitos identificados.

En la escuela, la psicóloga se encuentra en un constante ir y venir entre dos espacios. Por un lado, está lo que se ha denominado en este artículo "la periferia de la escuela", que corresponde a un espacio externo a los procesos pedagógicos que en ella ocurren; ahí se encuentra la psicóloga cumpliendo una función de staff de apoyo. Una de las características de este tipo de función es que no existe mayor involucramiento con la comunidad a la que se está prestando el servicio, trabajando en el tema convivencia en su oficina y en talleres, con el estudiantado señalado con "problemas de comportamiento". Esta condición periférica, como prestadora de servicios, se ve reforzada a causa de la situación contractual de la profesional, quien no posee un contrato de trabajo, sino un contrato de honorarios regido por el código civil en Chile. De este modo, el contexto de precarización laboral estaría condicionando un determinado escenario de relaciones sociolaborales en la escuela, atravesado por una sensación de temor de parte de la psicóloga.

Pero ¿qué pasa? la normativa te dice que si estás cumpliendo con un horario y tienes una continuidad laboral es contrato de trabajo, pero también pasa que uno como profesional dice "ya, me voy a poner como a mover", y ¿qué puede pasar?: "señorita, ya no necesitamos sus servicios"

En Chile, los contratos entre personas autónomas y organismos públicos o privados, son denominados "contratos de prestación de servicios" y la legislación los contempla como acciones esporádicas que no se someten a relación de subordinación y dependencia, tratándose de acciones transitorias y de apoyo. En este escenario, el hecho de que una buena parte del trabajo de esta psicóloga se encuentre restringido a la realización de talleres, y confinado en un espacio alejado de los demás profesionales, es interpretado por ella como una consecuencia del tipo de contrato que tiene.

Esto ha configurado un trabajo altamente individualizado y encapsulado, que debe dar respuesta a las demandas focalizadas sobre el estudiantado desde una posición de apoyo. Esto ha generado una distancia con el profesorado, enmarcándose en un escaso contacto cotidiano con éstos. A este aislamiento, producto en parte de la 
precarización, se le agrega la falta de articulación, la cual se describió en la categoría anterior, tal como señala el siguiente extracto de una profesora de aula.

Sí, de repente como muy aislado. I gual hay como que estar: "la psicóloga, la psicóloga, sal de tu Baticueva". Yo encuentro que es aislado y encuentro que falta articulación. Lamentablemente los tiempos no se nos dan, no hay tiempo para eso, no se dan para eso.

Esta baja articulación se encuentra mediada, además, por las condiciones de un sistema educativo que intensifica los tiempos de trabajo de todos sus actores, siendo el profesorado los más afectados como es percibido por el director de la escuela. Éste señala que diferentes programas gubernamentales como el Plan de Apoyo Compartido (PAC) intensifican los tiempos de trabajo, y exigen la rendición de cuentas, confirmando así la existencia de una cultura de la auditoría.

El tema es que estamos con éste, el otro, con la planificación y más encima nosotros tenemos PAC, tenemos, vigilancia, supervisión y tenemos que hacer muchas cosas aparte de educar

Esta intensificación, producto de políticas estandarizadas, generan un ambiente general de exigencia en relación a la rendición de cuentas que es reconocido por el profesorado tal como muestra a continuación una profesora de aula

Antiguamente al profesor no le exigían todas las semanas planificaciones, no le exigían todo, que tuviera las notas puestas tal fecha. No le exigían al profesor que sea evaluado, o que entre otra persona y te venga a ver la clase, ¿me entiendes?

En este escenario, la condición de staff de apoyo de la psicóloga se genera en la medida en que, desde este lugar, colabore con los procesos de control propios del sistema educativo, sometiéndose también al sistema de rendición de cuentas interno a través de la entrega de evidencias como confirma la jefa de UTP. En este sentido, la psicóloga comenta las exigencias que debe enfrentar en su trabajo que son percibidas como poco flexibles ya que no se le permite proponer nuevas acciones que no se encuentren presentes en el Plan de Mejoramiento Escolar. 
Yo necesito la evidencia de lo que ella hace, entonces ella hace la bitácora, un registro de con los niños que trabaja.

Ya, perfecto, si al final uno está como para prestar un servicio y obviamente está el hecho de que puedas servir en lo que es la necesidad del momento. Pero: - Sí, miren, igual podríamos hacer esto otro. Y responden: - No, pero es que no está en el plan de mejora.

Desde la micropolítica, se puede ver que se genera una tensión que responde a las jerarquías en el cual la jefa de UTP delimita a la psicóloga su espacio de acción confirmando el sistema de rendición de cuentas. Esta sensación de ser prestadora de un servicio que le exige la presentación de evidencias y que le otorga escasa flexibilidad le dificulta a la psicóloga identificarse como "la psicóloga de la escuela". En este sentido, lo que la psicóloga describe de forma implícita es que la exigencia de evidencia es una expresión de subordinación, y la dificultad para flexibilizar sus acciones implica que no posee efectivamente la autonomía de un trabajador a honorarios que realiza una asesoría independiente.

Como consecuencia de estas condiciones que la han relegado a lo que hemos denominado como "periferia", la psicóloga ha adoptado progresivamente una actitud de resignación, adecuándose a las exigencias y expectativas de los diversos actores educativos, dejando de lado sus proyectos personales-profesionales, aunque posee conciencia de esta situación, visibilizando en su discurso que el profesorado es un "grupo cerrado".

Adecuándome a lo que querían los profesores, sabiendo que a lo mejor no era lo mejor, o lo que yo hubiese querido hacer, pero tenía que cumplir con necesidades que en ese momento me estaban diciendo [...]. Hay reuniones en las cuales yo no participo, en algunas decisiones de las cuales yo no participo, porque los profesores tienen un grupo bien cerrado, entonces cuesta bastante entrar y yo, a pesar que llevo desde el 2010 trabajando, igual me ha costado.

Con el fin de llevar a cabo acciones vinculadas con su quehacer, la psicóloga va generando alianzas con las autoridades dentro de la escuela, y aunque esto confirma su rol "periférico", le permite administrar una cuota de poder que utiliza para generar actividades con cierta celeridad. 
Ustedes saben que también influye el tema de las personalidades. Obviamente uno no se puede llevar como al 100 \% con toda la gente, yo me siento más cercana al director. Entonces a lo mejor eso también influyó en que conseguía las cosas más rápido.

\section{Conclusiones y discusiones}

Esta investigación permitió analizar elementos macro, meso y micro sociales de construcción de identidad de una psicóloga escolar. Dentro de los elementos macro, se analizó la implementación de una política educativa que brinda incentivos a escuelas con población estudiantil en situación de pobreza para la mejora de resultados. Dentro de las medidas para lograr las metas de mejora fue contratada una psicóloga para realizar talleres y atención de estudiantes con "problemas de comportamiento". En este punto, se encontró un segundo elemento macro, que corresponde a la complicidad ideológica entre psicología y educación (Bahía Bock, 2003), en donde los problemas escolares son explicados por factores externos a la institución. En este caso, corresponde a la psicología individual de algunos estudiantes. La interacción de ambos elementos macro configuró su labor a distancia del quehacer de los profesores, con condiciones laborales precarias e intensificación del trabajo. La situación revela una paradoja que hace parecer que el trabajo entre la psicología y la educación se distancia, cuando en realidad este alejamiento es el que permite que la psicología sólo confirme el lugar del estudiantado sin favorecer procesos de reflexión pedagógica que permitan atribuciones internas de responsabilidad.

Esta condición de distancia estaba acoplada con la intensificación del trabajo también del profesorado, que en este estudio acusa no tener tiempo para articularse con la psicóloga, dadas las exigencias de cobertura curricular, supervisiones y evaluaciones de desempeño dispuestas por las políticas nacionales. Hypólito, Vieira y Pizzi (2009) conciben la intensificación del trabajo docente como un proceso en que los profesores responden a presiones crecientes y a innovaciones que se establecen sobre las mismas condiciones de trabajo, o incluso, en condiciones de precarización creciente. Se podría hipotetizar, a partir de los hallazgos de este estudio, que esta intensificación es utilizada como argumento para no articular el trabajo de la psicóloga con el de los docentes, permitiendo así que se siga señalando al estudiantado como responsable de sus aparentes desviaciones.

Hargreaves (1998) indica que, dentro de las características del proceso de intensificación del trabajo docente, se encuentra la escasez de tiempo para la actualización de contenidos y la calificación en habilidades necesarias, junto con el incremento del 
ingreso de especialistas a la escuela. Como se observa en este caso, desde un espacio micro social, la psicóloga es representada en el lugar de especialista en abordar problemas de conducta de los estudiantes. La metáfora de "la baticueva" expresada por una profesora, ilustra la distancia percibida con este campo de trabajo, dibujando el abordaje del comportamiento y la "disruptividad" del estudiantado en un espacio oscuro, confinado, con tecnologías sofisticadas y ajenas al cotidiano de la escuela.

Esta situación micro social se sostiene en una actitud de resignación de la psicóloga, quien se adecúa a las exigencias y expectativas de los diversos actores educativos, a pesar de ser consciente que estos no coinciden con sus ideales personales y profesionales. Esta resignación da cuenta de su complicidad en los términos de Bahía Bock (2003). Por una parte, señala estar entrampada (López, et al. 2011) por sus condiciones laborales, en las cuales su contrato de trabajo le asigna un rol clínico, el cual es demandado por el profesorado y controlado por la jefa de UTP. Indica que diversificar sus estrategias de intervención podría significar un descontento con su desempeño, arriesgando su despido. En este escenario, intersectan el nivel macro y micro de construcción de identidad, por cuanto es la propia política pública la que favorece las medidas de presión y el temor de la psicóloga, ya que la Ley SEP permite entonces este tipo de contrato civil.

Por otra parte, está el componente micro político, por cuanto la psicóloga indica que los profesores se constituyen como un "grupo cerrado". Frente a ello se observan escasas herramientas en ella para generar conversaciones y alianzas psicopedagógicas que contribuyan a movilizar esta situación. En el mismo plano micro político, la psicóloga expresa que sí encuentra afinidad con el director de la escuela y que a partir de ese vínculo puede conseguir con mayor facilidad lo que necesita para trabajar. Generar alianzas con la dirección de la escuela puede contribuir a encontrar legitimidad dentro de la comunidad escolar (Bardisa, 1997; Gómez, 2010). Sin embargo, al constituir un vínculo con la autoridad, puede generar resistencias o rechazo desde el cuerpo de profesores, quienes disputarían su propio espacio de autonomía y poder dentro de la escuela (Gómez, 2010). De este modo, la legitimación desde la autoridad que podría encontrar la psicóloga en su cercanía con el director, si bien pueda brindarle validación a su rol dentro de la institución, a su vez reforzaría su rol periférico respecto del profesorado.

Los resultados no pretenden la generalización. Sí buscamos aportar con conocimientos sobre la relación entre la macro y la micropolítica con la construcción del rol y la identidad del psicólogo en la escuela. Lo que el estudio evidenció, llama a cuestionar las políticas de empaquetamiento curricular y de control del desempeño docente, que inciden en intensificar su trabajo, dificultando el abordaje de lo que queda fuera 
del paquete: lo disruptivo y lo diverso. Buscando subsanar esta situación, se erige una política de discriminación positiva - Ley SEP-, que facilita la entrada de psicólogos y otros profesionales a la escuela. No obstante, su focalización en los estudiantes con diagnóstico de vulnerabilidad psicosocial o con problemas de comportamiento, junto con la supuesta especialización de su abordaje, apunta a sumar esfuerzos por mejorar los resultados e indicadores institucionales, antes que a fortalecer procesos interdisciplinarios y desarrollos profesionales en el ejercicio de los cargos.

Por último, la escasez de herramientas observadas tanto en la psicóloga como en el profesorado participante, llama la atención respecto de necesidades de formación en habilidades para comprender y transformar la organización y la cultura de la escuela. A través de la creación de lenguajes comunes cobraría sentido el aunar esfuerzos para construir abordajes complejos para los desafíos de la educación escolar de niños, niñas y jóvenes.

\section{Referencias}

Assaél, J., Contreras, P., Corbalán, F., Palma, E., Campos, J., Sisto, V. y Redondo, J. (2012). Ley SEP en escuelas municipales emergentes: ¿cambios en la identidad docente? Paulo Freire. Revista de Pedagogía Crítica, 11(11), 219-228 Recuperado de: http://revistas.academia.cl/index.php/ pfr/article/viewFile/453/594

Assaél, J.; Acuña, F.; Contreras, P. y Corbalán, F. (2014) Transformaciones en la cultura escolar en el marco de la implementación de políticas de Accountability en Chile: Un estudio etnográfico en dos escuelas clasificadas en recuperación. Estudios Pedagógicos, 40(2), 7-26. doi: https://dx.doi.org/10.4067/S0718-07052014000300001

Bahía Bock, A. (2003). Psicología de la Educación: Complicidad Ideológica. En A. Bock, A. Checcia y M. Rebello. (Eds.), Psicología Escolar: Teorías criticas (pp. 79-103). Sao Paulo, Brasil: Casa do Psicólogo.

Ball, S. (1994). La micro política de la Escuela. Hacia una teoría de la organización escolar. Madrid: Paidos.

Baltar, M. (2003). El sentido del diagnóstico psicológico escolar: Un análisis crítico y una propuesta en construcción. Psicoperspectivas, individuo y sociedad, 2, 7-34. Recuperado de: http://www.psicoperspectivas.cl/index.php/psicoperspectivas/article/view/1 
Baltar,M. J.yCarrasco,C. (2013). Re-pensando lapsicologíaeducacionalenChile:Análisiscrítico desu quehacery sugerencias proyectadas. Psicología para América Latina, 24, 173-190. Recuperado de: http://pepsic.bvsalud.org/scielo.php?script=sci_arttext\&pid=S1870-350X2013000100011

Bardisa, T. (1997). Teoría y práctica de la micro política en las organizaciones escolares. Revista Iberoamericana de Educación, 15, 13-52. Recuperado de: https://rieoei.org/historico/oeivirt/ rie15a01.htm

Barraza, R. (2015). Perspectivas acerca del rol del psicólogo educacional: propuesta orientadora de su actuación en el ámbito escolar, Revista Actualidades investigativas en educación, 15(3), 1-21. doi: http://dx.doi.org/10.15517/aie.v15i3.21070

Bellei, C. y García Huidobro, J. (2006). ¿Remedio para la inequidad? La subvención escolar preferencial. Revista Mensaje, 547, 1-7 Recuperado de: http://www7.uc.cl/facteo/centromanuellarrain/htm/garciahuidobro_remedio.htm

Bellei, C., Osses, A. y Valenzuela, J. P. (2010). Asistencia técnica educativa: de la intuición a la evidencia. Santiago de Chile, Chile: Salesianos.

Bolívar, A. y Ritacco, M. (2016) Identidad profesional de los directores escolares en España. Un enfoque biográfico narrativo. Opción, 32(79) 163-183. Recuperado de: http://www.redalyc. org/articulo.oa?id=31046684010

Canales, M (2006). Metodologías de Investigación Social: Introducción a los Oficios. Santiago: LOM Ediciones.

Castro, F.; Mazo, P. y Quintanilla, P. (2016). Instrumentos claves de la gestión escolar. El caso de la subvención escolar preferencial de chile. Actualidades Investigativas en Educación, 16(3), 97128. doi: https://dx.doi.org/10.15517/aie.v16i3.25958

Compagnucci, E. y Cardós, P. (2009). El desarrollo del conocimiento profesional del psicólogo: estudio de graduados de la UNLP, su inserción en el campo educativo. Orientación y sociedad, 9, 1-11. Recuperado de: http://www.scielo.org.ar/scielo. php?script=sci_arttext\&pid=S1851-88932009000100004

De Souza, C., Ribeiro, M. J. y Cintra da Silva, S. M. (2011). A atuação do psicólogo escolar na rede particular de ensino. Psicologia Escolar e Educacional, 15(1), 53-61. doi: http://dx.doi. org/10.1590/S1413-85572011000100006 
Donoso-Díaz, S. y Benavides-Moreno, N. (2017). Descentralización de la gestión de la educación pública e institucionalidad local en Chile: el caso de los directores comunales de educación. Innovar, 27(64), 115-128. doi: https://dx.doi.org/10.15446/innovar.v27n64.62372

Fernández, J.; Vázquez, M. y Ubiarco, A. (2016). Rol del psicólogo educativo. Una mirada desde sus actores, Revista Iberoamericana de Producción Académica y Gestión Educativa, 3(5), 1-15 Recuperado de: http://www.pag.org.mx/index.php/PAG/article/view/562/599

Fuentes, S. y Cruz, O. (2016). Micro política escolar y vida institucional en escuelas primarias de México. RIDE. Rev. Iberoam. Investig. Desarro. Educ 7(13), 345-369. Recuperado de: http:// www.scielo.org.mx/scielo.php?script=sci_arttext\&pid=\$2007-74672016000200345

García, C., Carrasco, G., Mendoza, M. y Pérez, C. (2012). Rol del psicólogo en establecimientos particulares pagados del Gran Concepción, Chile: Un proceso de co-construcción. Estudios Pedagógicos. 28(2), 169-185. doi: http://dx.doi.org/10.4067/S0718-07052012000200011

Gómez, A. (2010). Micro política escolar y procesos de cambio: el papel del supervisor en una institución educativa. Revista mexicana de investigación educativa, 15(46), 771-802. Recuperado de: http://www.scielo.org.mx/scielo.php?script=sci_arttext\&pid=S1405-66662010000300005

González, M., González, I. y Vicencio, K. (2014). Descripción del rol autopercibido del psicólogo y sus implicanciasen losprocesosdeformación depregrado. Psicoperspectivas, IndividuoySociedad, 13(1), 108-120. doi: http://dx.doi.org/10.5027/psicoperspectivas-Vol13-Issue1-fulltext-296

Hargreaves, A. (1998). Os professores em tempos de mudança - o trabalho e a cultura dos professores na idade pós-moderna. Lisboa, Portugal: Mc Graw-Hill.

Hypolito, Á. M., Vieira, J. S. y Pizzi, L. C. V. (2009). Reestruturação curricular e autointensificação do trabalho docente. Currículo sem fronteiras, 9(2), 100-112. Recuperado de: http://132.248.9.34/ hevila/CurriculosemFronteiras/2009/vol9/no2/6.pdf

Ibáñez, J. (1994) El Regreso del Sujeto: La Investigación Social de Segundo Orden. Madrid, España: Siglo XXI.

Íñiguez, L. (2001). Identidad de lo personal a lo social. Un recorrido conceptual. En E. Crespo, (Ed.) La constitución social de la subjetividad. Madrid, España: Catarata.

Juliá, M. T. (2006) Competencias profesionales del psicólogo educacional: una tarea asociativa. Revista de Psicología, 15(2), 115-130. Recuperado de: http://www.redalyc.org/articulo. oa?id=26415207 
Ligueño, S.; Palacios, D. y Sandoval, J. (2018). Discursos de la vulnerabilidad bajo el gerenciamiento educativo: Análisis Ley de subvención escolar preferencial chilena. Revista de Investigacion Psicologica, 19, 207-222. Recuperado de: http://www.scielo.org.bo/scielo. php?script=sci_arttext\&pid=\$2223-30322018000100014\&lng=es\&tlng=es.

López, V. y Carrasco, C. (2018) El/la psicólogo/a en educación: un análisis desde “lo psicosocial” y la convivencia escolar. En F. Leal. (Ed.), Psicólogos en la escuela: el replanteo de un rol confuso. Temas en Psicología Educacional. Contribuciones para la formación de especialidad. Buenos Aires, Argentina: Noveduc-Ensayos \& Experiencias-Universidad de Tarapacá-Edulnclusiva.

López, V., Carrasco, C., Morales, M. y Ayala, A. (2011) El encapsulamiento de los psicólogos escolares y profesionales de apoyo psicosocial en la escuela. Revista Internacional Magisterio, 53, 54-57.

Ministerio de Educación (MINEDUc) (2009). Ley de Subvención Escolar Preferencial. Un camino para mayor equidad. Revista Nuestros Temas, 29. Recuperado de: http://www.facso.uchile.cl/psicologia/epe/_documentos/GT_cultura_escolar_politica_educativa/recursos\%20 bibliograficos/material\%20mineduc/mineduc\%282009\%29informativoseppadresyprofesores.pdf

Ossa, C. (2011). El rol del Psicólogo Educacional: La transición desde el paradigma de la simplicidad al paradigma de la complejidad. Revista Pequén, I(1), 72 - 82. Recuperado de: http:// revistas.ubiobio.cl/index.php/RP/article/view/1820/1764

Palacio, J.; Correa, A.; Díaz, M. y Jiménez, S. (2003) La búsqueda de la identidad social: un punto de partida para comprender las dinámicas del desplazamiento - restablecimiento forzado en Colombia. Investigación \& Desarrollo, 11(1), 26-55. Recuperado de: http://www.redalyc.org/ articulo.oa?id=26811102

Pérez, M. (2016). La evaluación psicológica en contextos educativos: aciertos del pasado, errores del presente y propuestas de futuro. Estudos de Psicologia (Campinas), 33(3), 465-476. doi: https://dx.doi.org/10.1590/1982-02752016000300010

Prieto, M. (2001). La Investigación en el Aula: ¿Una tarea posible? Valparaíso, Chile: Universidad Católica de Valparaíso.

Pujal, M. (1996). La identitat (el self). En M. Botella et. al. (Comp.), Psicología Social (pp. 61-109). Barcelona, España: EDIUOC.

Quintana, A. (2006) Psicología: Tópicos de actualidad. Metodología de Investigación Científica Cualitativa. Lima: UNMSM. 
Raczynski, D.; Muñoz, G.; Weinstein, J., y Pascual, J. (2013) Subvención escolar preferencial (SEP) en Chile: un intento por equilibrar la macro y micro política escolar REICE. Revista Iberoamericana sobre Calidad, Eficacia y Cambio en Educación, 11(2), 164-193 Recuperado de: http://www. redalyc.org/pdf/551/55127024008.pdf

Rojas, M.T. y Leyton, D. (2014) La nueva subjetividad docente: Construcción de subjetividades docentes en los inicios de la implementación de la Subvención Escolar Preferencial en Chile. Estudios pedagógicos, 40 (No.Especial), 205-221. Recuperado de: http://mingaonline. uach.cl/scielo.php?script=sci_arttext\&pid=S0718-07052014000300012\&lng=es\&nrm=iso

Rodríguez, E. (2016) Micro política escolar y el liderazgo directivo en la escuela. Revista Educación, 41(1), 1-14. doi: https://doi.org/10.15517/revedu.v41i1.21407

Rodríguez, G., Gil, J. y García, E. (1996) Metodología De La Investigación Cualitativa. Granada, España: Ediciones Aljibe.

Salamanca, A. y Martín-Crespo, C. (2007) El muestreo en la investigación cualitativa. Nure Investigación, 27, 1-4. Recuperado de: http://www.nureinvestigacion.es/OJS/index.php/ nure/article/view/340/330

Sandoval, E. y Lamas, M. (2017) Impacto de la Ley SEP en las escuelas: Una mirada crítica y local en torno al rol de los psicólogos de la educación, Paideia, 61, 57-81. Recuperado de: http://www.revistapaideia.cl/index.php/PAIDEIA/article/view/170/131

Tajfel, H. y Turner, J. (1979). An integrative theory of intergroup conflict. En S. Worchel and W. Austin. (Eds.), The social psychology of intergroup relations. Monterey: Brooks-Cole.

Vázquez, F. (1994). Análisis de contenido categorial: el análisis temático. Barcelona, España: Universitat Autónoma de Barcelona.

Vera-Bachmann, D.; Valenzuela, A. y Álvarez-Espinoza, A. (2016). Psicología Educacional en contextos rurales: ¿estamos preparados? Notas acerca del caso chileno. Rev. Int. Investig. Cienc. Soc., 12 (2), 283-302. doi: https://dx.doi.org/10.18004/riics.2016.diciembre.283-302

Vera-Bachman, D. y Salvo, S. (2016). Perfiles de escuelas rurales exitosas: Una propuesta de la psicología educacional ante el cierre de escuelas. Universitas Psychologica, 15(2), 25-38. doi: http://dx.doi.org/10.11144/Javeriana.upsy15-2.pere 
Verger, A., Bonal, X. y Zancajo, A. (2016). Recontextualización de políticas y (cuasi)mercados educativos. Un análisis de las dinámicas de demanda y oferta escolar en Chile. Education Policy Analysis Archives/Archivos Analíticos de Políticas Educativas, 24, 1-27

Zibas, D. (1997) ¿Un juego de espejos rotos? La vida escolar cotidiana y las políticas educativas en América Latina Revista Iberoamericana de Educación, 15, 121-137. Recuperado de: https:// rieoei.org/historico/oeivirt/rie15a05.htm 